\title{
Model Predictive Control
}

\author{
Baocang Ding, ${ }^{1}$ Marcin T. Cychowski, ${ }^{2}$ Yugeng Xi, ${ }^{3}$ Wenjian Cai, ${ }^{4}$ and Biao Huang ${ }^{5}$ \\ ${ }^{1}$ Ministry of Education Key Lab For Intelligent Networks and Network Security (MOE KLINNS Lab), \\ Department of Automation, School of Electronic and Information Engineering, Xi'an Jiaotong University, Xi'an 710049, China \\ ${ }^{2}$ NIMBUS Centre for Embedded Systems Research, Cork Institute of Technology, Rossa Avenue, Cork, Ireland \\ ${ }^{3}$ Department of Automation, Shanghai Jiaotong University, Shanghai 200240, China \\ ${ }^{4}$ School of Electronic and Electrical Engineering, Nanyang Technological University, BLK S2, Singapore 639798 \\ ${ }^{5}$ Department of Chemical and Materials Engineering, University of Alberta, Edmonton, AB, Canada T6G $2 G 6$
}

Correspondence should be addressed to Baocang Ding, baocang.ding@gmail.com

Received 9 February 2012; Accepted 9 February 2012

Copyright ( 2012 Baocang Ding et al. This is an open access article distributed under the Creative Commons Attribution License, which permits unrestricted use, distribution, and reproduction in any medium, provided the original work is properly cited.

Model predictive control (MPC) has been a leading technology in the field of advanced process control for over 30 years. At each sampling time, MPC optimizes a performance cost satisfying the physical constraints, which is initialized by the real measurements, to obtain a sequence of control moves or control laws. However, MPC only implements the control move corresponding to the current sampling time. At the next sampling time, the same optimization problem is solved with renewed initialization. In order to obtain offset-free control, the system model is augmented with a disturbance model to capture the effect of model-pant mismatch. The state and disturbance estimates are utilized to initialize the optimization problem. A steady-state target calculation unit is constructed to compromise between what is desired by real-time optimization (RTO) and what is feasible for the dynamic control move optimization. As a result, MPC exhibits innumerous research issues from both theoretical and practical aspects. Nowadays, there is big gap between the theoretical investigations and industrial applications. The main focus of this special issue is on the new research ideas and results for MPC.

A total number of 14 papers were submitted for this special issue. Out of the submitted papers, 6 contributions have been included in this special issue. The 6 papers consider 6 rather different, yet interesting topics.

A synthesis approach of MPC is the one with guaranteed stability. The technique of linear matrix inequality (LMI) is often used in the synthesis approach to address the satisfaction of physical constraints. However, the existing LMI formulations are for symmetric constraints. M. S. M. Cavalca et al. treat asymmetric output constraints in integrating SISO systems based on pseudoreferences.

Usually, the steady-state target calculation, followed with the dynamic move calculation, is implemented in each sampling time. This is not practical for fast-sampling systems. Y. C. Chu and M. Z. Q. Chen propose a method to tackle this issue. In their method, the steady-state target calculation works in parallel to, with a period longer than and a scale of optimization larger than, the dynamic move calculation. It is shown that their method is particularly suitable for tracking the periodic references.

The nonlinear system represented by a Hammerstein model has always been a good platform for control algorithm research. D. F. He and L. Yu revisit this topic by invoking the pole-placement method on the linear subsystem. They propose the algorithm which consists of three online steps, instead of the two-step MPC. They also propose to use their algorithm in the grade transition control of industrial polypropylene plants, via a simulation study.

Usually, MPC has its own paradigm for robust control. However, combing MPC with $\mathscr{H}_{2} / \mathscr{H}_{\infty}$ control could be a good topic for improving the robustness of MPC. P. E. Orukpe applies the mixed $\mathscr{H}_{2} / \mathscr{H}_{\infty}$ method in MPC where the system uncertainties are modeled by perturbations in a linear fractional transform (LFT) representation and unknown bounded disturbances.

Interests in the cooperative control of multi-agent systems have been growing significantly over the last years. MPC has the ability to redefine cost functions and constraints as needed to reflect changes in the environment, which makes 
it a nice choice for multiagent systems. S. B. Wei et al. give a method for distributed MPC which punishes, in the cost function, the deviation between what an agent optimizes and what other related agents think of it. The deviation weight matrix at the end of the control horizon is specially discussed for improving the control performance.

Besides, C. H. F. Silva et al. report some experimental studies for two classical algorithms: infinite horizon MPC and MPC with reference system. The pilot plant is for level and $\mathrm{pH}$ control, which has physical constraints and nonlinear dynamics.

We hope the readers of Journal of Control Science and Engineering will find the special issue interesting and stimulating, and expect that the included papers contribute to further advance the area of MPC.

\section{Acknowledgments}

We would like to thank all the authors who have submitted papers to the special issue and the reviewers involved in the refereeing of the submissions.

Baocang Ding

Marcin T. Cychowski

Yugeng Xi

Wenjian Cai

Biao Huang 

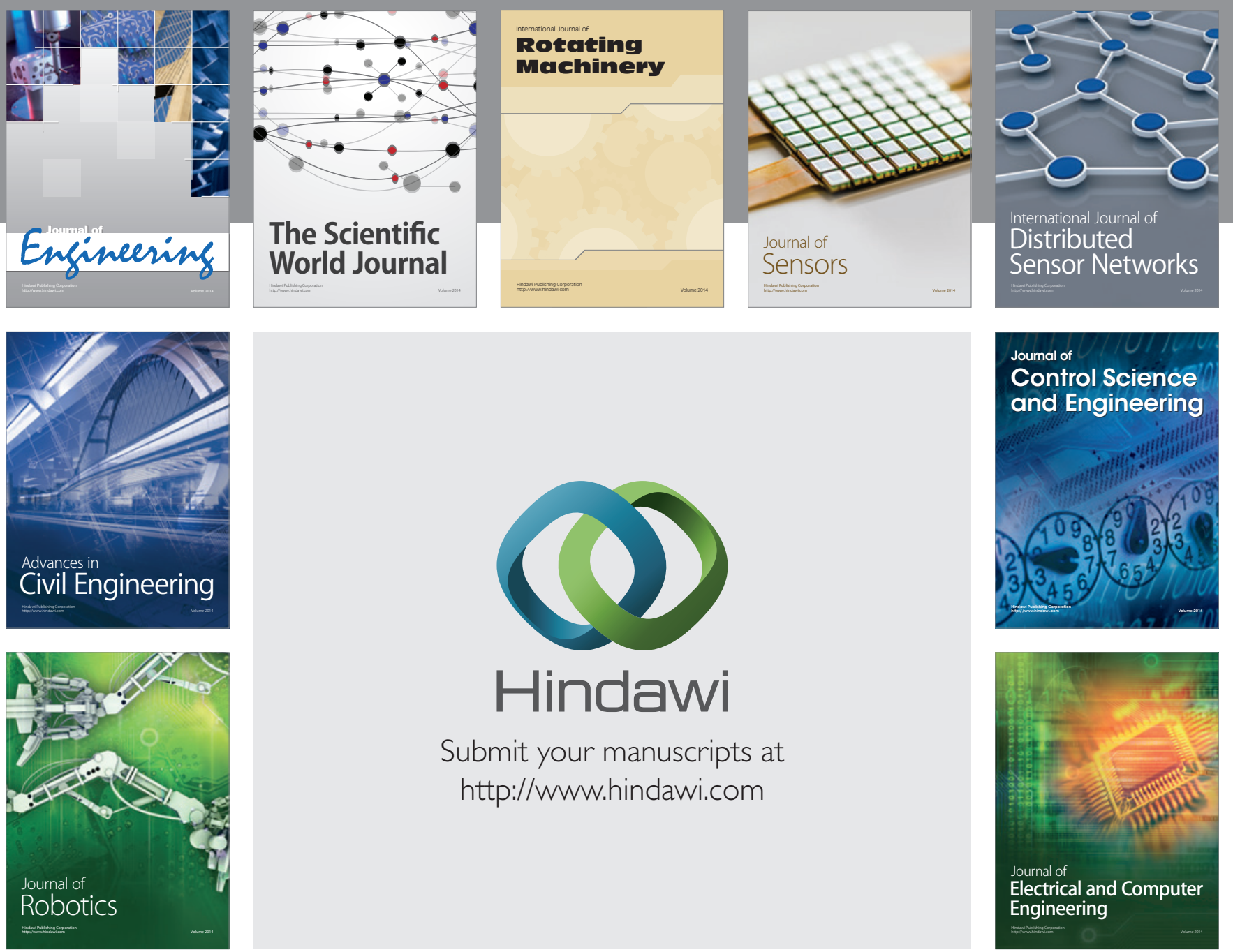

Submit your manuscripts at

http://www.hindawi.com
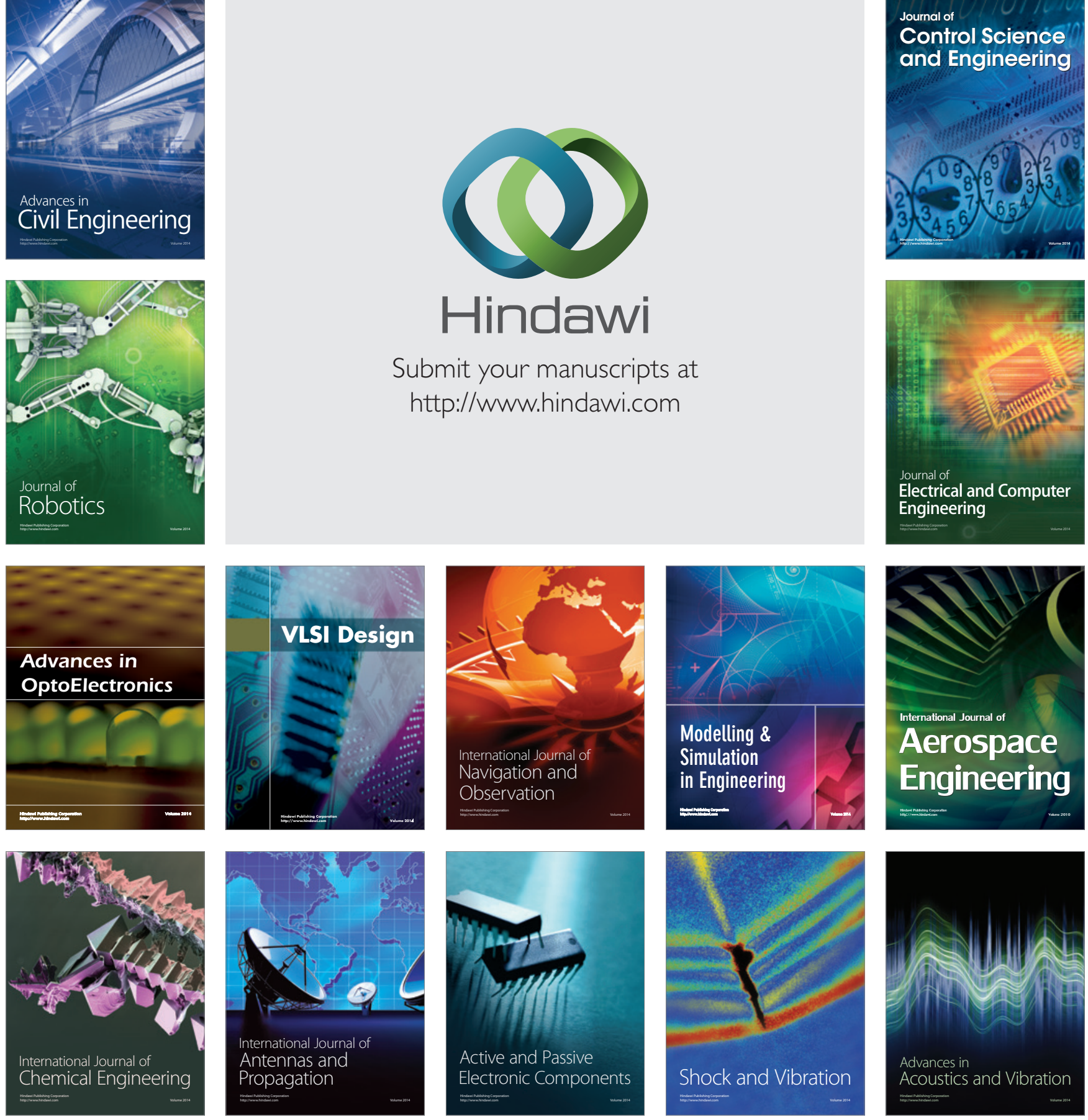